\title{
Assessment of health risks of policies
}

Balázs Ádám ${ }^{1,2 *}$, Ágnes Molnár ${ }^{2,3}$, Róza Ádány ${ }^{2}$, Fabrizio Bianchi ${ }^{4}$, Katarina Bitenc ${ }^{5}$, Razvan Chereches ${ }^{6}$, Liliana Cori ${ }^{4}$, Rainer Fehr ${ }^{7}$, Joanna Kobza ${ }^{8}$, Jana Kollarova ${ }^{9}$, Stella R. J. Kræmer $^{1}$, Nunzia Linzalone ${ }^{4}$, Marek Majdan ${ }^{10}$, Odile Mekel ${ }^{7}$, Peter Mochungong ${ }^{1}$, Peter Otorepec $^{5}$, Jozef Pastuszka ${ }^{11}$, Sarah Sierig ${ }^{7}$, Ingrida Zurlyte ${ }^{12}$, Gabriel Gulis ${ }^{1}$

${ }^{1}$ Unit for Health Promotion Research, Faculty of Health Sciences, University of Southern Denmark, Niels Bohrs Vej 9, DK-6700 Esbjerg, Denmark, ${ }^{2}$ Department of Preventive Medicine, Faculty of Public Health, Medical and Health Science Centre, University of Debrecen, P.O. Box 9, H-4012 Debrecen, Hungary, ${ }^{3}$ Centre for Research on Inner City Health, Keenan Research Centre Li Ka Shing Knowledge Institute, St Michael's Hospital, Victoria 209, Rm. 3-26.22, M5B 1C6 Toronto, Ontario, Canada, ${ }^{4}$ Unit of Environmental Epidemiology, Institute of Clinical Physiology, National Council of Research, Moruzzi 1, 56124 Pisa, Italy, ${ }^{5}$ National Institute of Public Health, Trubarjeva 2, SI-1000 Ljubljana, Slovenia, ${ }^{6}$ Faculty of Political, Administrative and Communication Sciences, Babes-Bolyai University, Strada Mihail Kogalniceanu 1, 3400 Cluj, Romania, ${ }^{7}$ Regional Health Center North-Rhine Westphalia, Gesundheitscampus 9, 44801 Bochum, Germany, ${ }^{8}$ Public Health Department, Silesian Medical University, 18 Medykow Street, 40-752 Katowice, Poland, ${ }^{9}$ Department of Health Promotion, Regional Public Health Authority, Ipelska 1, 04011 Kosice, Slovakia, ${ }^{10}$ Department of Public Health, Faculty of Health and Social Work, Trnava University, Univerzitne namestie 1, 91701 Trnava, Slovakia, ${ }^{11}$ Department of Air Protection, Faculty of Energy and Environmental Engineering, Silesian University of Technology, Akademicka 2A, 44-100 Gliwice, Poland, ${ }^{12}$ Centre for Health Education and Disease Prevention, Kalvariju 153, LY-08221 Vilnius, Lithuania

*Corresponding author: Balázs Ádám, Centre of Maritime Health and Society, Faculty of Health Sciences, University of Southern Denmark, Niels Bohrs Vej 9, DK-6700, Esbjerg, Denmark, tel: 4565504203, fax: 4565501091, email: badam@cmss.sdu.dk 


\begin{abstract}
The assessment of health risks of policies is an inevitable, although challenging prerequisite for the inclusion of health considerations in political decision making. The aim of our project was to develop a so far missing methodological guide for the assessment of the complex impact structure of policies. The guide was developed in a consensual way based on experiences gathered during the assessment of specific national policies selected by the partners of an EU project. Methodological considerations were discussed and summarized in workshops and pilot tested on the EU Health Strategy for finalization. The combined tool, which includes a textual guidance and a checklist, follows the top-down approach, that is, it guides the analysis of causal chains from the policy through related health determinants and risk factors to health outcomes. The tool discusses the most important practical issues of assessment by impact level. It emphasises the transparent identification and prioritization of factors, the consideration of the feasibility of exposure and outcome assessment with special focus on quantification. The developed guide provides useful methodological instructions for the comprehensive assessment of health risks of policies that can be effectively used in the health impact assessment of policy proposals.
\end{abstract}

Keywords: Risk assessment; Health impact assessment; Policy making; Health in all policies 


\section{Introduction}

Health is determined by a wide range of factors, most of them falling outside the scope of the health sector. Because health determinants represent virtually all areas of life, a political decision very likely affects health. The aim of conducting healthy public policy appears as an action area in the Ottawa Charter for Health Promotion followed by the Adelaide Recommendations on Healthy Public Policy. Both documents emphasize the need of political commitment for promoting health at the population level (WHO, 1986; WHO, 1988). The inclusion of human health and well-being as one of the main principles of policy development in all sectors is the concept of Health in All Policies (Stahl et al., 2006; WHO and Government of South Australia, 2010). However, it is not an easy task since evidence based consideration of health issues is not readily accommodated in the policy process of non-health sectors even in areas closely related to health, though it can be substantially facilitated by appropriate methodology.

Health impact assessment (HIA) is a methodological framework for predicting health impact of policy proposals and consequently to assist decision makers (WHO European Centre for Health Policy, 1999), although its incorporation in the decision-making process is cumbersome (Davenport et al., 2006; Mannheimer et al., 2007; Ádám, 2012). The standard HIA methodology consisting of screening, scoping, risk appraisal, decision making, monitoring and evaluation works well on projects and programs where well-defined hazards are identified. The involvement of all stakeholders, combination of both qualitative and quantitative approaches and use of risk assessment methodology provides sound tool to quantify the risks related to hazards. If HIA is applied to policies a similar risk assessment method which would allow identification and quantification of risks is missing. Methods exist to quantify health effects in the risk factor-health outcome relation (Steenland and Armstrong, 
2006; Lhachimi et al., 2010; Manuel et al., 2012; Lhachimi et al, 2012), especially the effects of environmental factors acting in a single causal pathway (Basham, 2001; McCarthy et al., 2002; Utley et al., 2003; Mesa-Frias et al., 2013). A concise, applicable guide that aids the impact assessment of the complex causal web of policies including social determinants of health is, however, not yet available.

The recognition of this deficiency in knowledge and methodology for conducting risk assessment on policy health effect led to the launch of the Risk Assessment from Policy to Impact Dimension (RAPID) 2009-2012 EU-funded project (RAPID, 2012). It had the primary aim to develop a methodological guidance for the assessment of health risks related to policies that analyse the "full chain" of causal pathways from policy level through health determinants and risk factors to health outcomes in an integrated manner. This article introduces the product of a series of methodological findings that provides a useful tool for either the HIA community or for those engaged with the assessment of policies, enabling them to effectively assist the political decision-making process regardless of the level and sector of the policy proposal. 


\section{Methods}

The guidance for the top-down approach of assessing health risks of policies in an integrated manner was elaborated in a consensual way in the RAPID project. Ten partners from universities and national public health institutions of nine EU countries participated in the work. Each project partner selected a specific national policy and carried out its assessment. They could freely decide on the application of adequate methods and national and international information sources, only the main steps of the assessment were determined in advance. The assessment meant to analyse the policy and to describe all wider, including socio-economic, determinants of health influenced by the policy. Risk factors linked to these health determinants and health outcomes related to selected risk factors were finally identified and assessed. A strong focus of the project was to include quantitative risk assessment techniques in the analysis. The guidance was formulated with recommendations on various aspects of conducting quantitative assessment, like information need and feasibility, integration of quantitative and qualitative assessment elements in specified pathways, evaluation of horizontal interactions between elements of the same impact level, and characterization of uncertainty.

The case studies provided the basic input for the development of the guidance. The methodological findings, experiences and considerations that were found useful in the preparation of the case studies were presented and discussed on several work meetings of the project and the most expedient elements of the consensual methodology were summarized. After the consensus discussion the developed tool was circulated for final comments and approved as a working version. The first version of the agreed guidance was used to analyse selected parts of the EU Health Strategy (2008-2013). The experiences of the pilot testing allowed for its further improvement. The tool was then presented on a series of national 
workshops that were organized for experts involved in various risk assessment practices in the participating countries. The participants were invited from a database of risk assessors that was constructed in a previous phase of the project. They represented diverse areas (health, economics, construction, etc.) and organisations (academic, governmental, private). The expert opinion of participants provided feedback to finalize the guidance as well as opportunity to distribute it in the professional community of risk assessors as potential users. 


\section{Results}

\subsection{Guidance for the assessment of health risks of policies}

\subsubsection{Policy}

The assessed policy (strategy, program or regulation) is preferably of substantial importance from a population health point of view, regardless of the sector and level of its initiation that can be either central, regional or local government, industry or other organizations. The commission for the conduct of assessment can come from policy makers. If the tool is applied within an on-going HIA the task can come from the scoping part of the process; if not used within an HIA than all following issues need to be considered at the beginning of assessment. In case a policy is not given at forehand or the policy is too broad that should be narrowed down, a transparent selection process is important. The most appropriate way is to have a set of criteria for the pre-selection of policies that should include the importance of the topic and the need of policy makers for assistance in the decision-making process. It is recommended to analyse the pre-selected policies by feasibility issues like availability of verifiable objectives, definable target group, valid data and dose-response functions and evidence on influenced health determinants and risk factors.

After the policy to be assessed is defined, a good understanding of the policy context is of crucial importance in order to be able to link the policy to impacts on health and health inequalities: map the impact structure, prioritize impact pathways and identify where the challenges come from. At first, problem identification and demand for action should be described in a concise way. Placing a national policy into an international context helps to identify the driving forces of policy making, meanwhile allows for finding similar policies implemented in other countries the experiences of which can be fruitfully applied then in the 
assessment process. It is worth reviewing the history of the policy, how it developed with time. The understanding of the legal environment and the relationship between the assessed and other related policies allows for the consideration of their interactions. The construction of a map about policy`s context in the national and international legal environment can be useful. The policy context regarding stakeholders and interests should be described in order to explain the development and main drivers of the policy in an objective manner. Detailed description of the policy content is needed in order to demonstrate the main goals of the policy, tools of implementation and methods of monitoring and evaluation if it is relevant. Force and effect of the legislation should be examined in order to identify target population and time course of the implementation. A policy can be well prepared, however, its implementation rather poor. To be able to assess the predictable effects of a policy, the feasibility of its measures should also be taken into account; the complexity of implementation, availability of resources (institutional, human and financial) as well as public acceptance and compliance should be all considered. A policy that has clear implementation plan is easier to be assessed for feasibility. The costs involved in implementation gives a starting point to cost-benefit considerations. The analysis of different scenarios, including the status quo, can effectively assist the choice between various policy options.

\subsubsection{Health determinants}

Health determinants are to link policies to the direct risk factors of health in the assessment process. In this way, the factors that are considered as the determinants - or wider determinants, upstream determinants, causes of causes - of health have typically rather qualitative nature in the assessment process. Determinants of health are defined by the Health promotion glossary of WHO as "the range of personal, social, economic and environmental factors which determine the health status of individuals or populations" (WHO, 1998). In 
some cases, determinants of health might overlap with risk factors and it is difficult to differentiate them. Using a model that provides a set of health determinants can help to overcome this problem. There are various models to describe the structure of health determinants; those presenting the holistic model of health are preferable. The model of Lalonde can be effectively used (Lalonde, 1974), but other scientifically recognized models, like that of Dahlgren and Whitehead (Dahlgren and Whitehead, 1991), or Barton and Grant (Barton and Grant 2006) can also be applied.

The ways for identifying policy-influenced health determinants can vary, therefore the selection method should be transparently described. It can include extensive literature review, expert opinion or even involvement of stakeholder participation. The key issue in the selection of influenced health determinants is the availability of evidence for causality. Two approaches of selection can be distinguished: the broad consideration (not to lose any) and the strict one that prioritises health determinants. Prioritization should focus on the strength of evidence available and feasibility of - favourably quantitative - assessment of health outcomes through the assessment of changes in the exposure levels of risk factors. Making decisions on an impact level based on the anticipatory evaluation of priority and feasibility issues related to lower levels, e.g. avoiding the selection of a health determinant to which risk factors and health outcomes cannot be related downstream in the impact pathway due to lack of evidence for causality, is a beneficial strategy for effective prioritisation. It can be referred to as internal loops of consideration between the levels of the causal chain. The selection process also depends on the primary intention of the assessment, that is, what policy-makers want to use the assessment for. Furthermore, the amount of resources available determines the need for prioritisation, too.

To conclude, a clear strategy for choosing health determinants for assessment is necessary. If selection is to be narrowed down, the way of prioritizing among the various pathways should 
consider the strength of available evidence for causality that enables the judgement on the likelihood of the existence of impact. The other crucial aspect of prioritization is the size/importance of the effect (size of population affected, severity of health effects, costs involved etc.). The formalization of the prioritization process by using a systematic approach to assign values to the elements of causal chains by context-specific aspects of priority can further increase transparency.

\subsubsection{Risk factors}

Risk factors are those factors of the impact chain that directly affect health (proximal factors). Risk factors are defined according to the Health Promotion Glossary of WHO as "social, economic or biological status, behaviours or environments which are associated with or cause increased susceptibility to a specific disease, ill health, or injury" (WHO, 1998). This definition specifies factors with negative effect on health. However, one has to be aware that a factor could also be a protective factor having a positive effect on health. The prevalence or level of their contact with individuals is referred to as exposure. It is imperative to make a distinction between the risk factors acting in the impact chain and the risk factors modifying individual susceptibility. The latter is to be considered when identifying susceptible subgroups of the affected population.

To be able to assess the possible health outcomes of the selected causal pathways, thorough review and enlistment of all influenced risk factors is indispensable. The assessment should describe the exposed population, the routes of exposure and the exposure pattern in different population groups. The latter enables specific assessment of effects on various groups that allows for considering equity issues. The prioritization of risk factors is based essentially on the same principles as that of health determinants. It should take into account the strength of evidence for causality, reliability of literature source and biological plausibility, as well as the 
significance of induced health effects. The latter is determined by the size of exposure change attributable to the policy, size of population affected and severity of related health outcomes. Since the extent of being exposed to risk factors may be characterized not only qualitatively but measured in a quantitative way, an important consideration of selection for detailed analysis is the feasibility of numerical explanation. The quantification of exposure change due to policy implementation is based on the availability of applicable exposure measures and numerical information on the baseline level/prevalence of exposure, as well as on the expected change of exposure related to policy implementation (exposure assessment). The latter is ideally provided by studies observing the consequences of the introduction of similar policies, or can be based on expert opinion which usually implies larger uncertainty. The demand for quantification also depends on the interest of policy-makers and other stakeholders. Quantitative exposure assessment can provide the basis for the quantification of health outcomes, therefore availability of valid data and dose/exposure-response coefficients for health outcome assessment should already be considered in the selection process of risk factors, making internal loops of consideration between the levels of the causal chain.

\subsubsection{Health outcomes}

Health outcomes that are causally related to the identified risk factors represent the final consequences of impact pathways. They must be unambiguously defined, since various stages of a causal chain can be considered as outcomes, like a disease or health states and events related to a disease. An adequate solution for clarification is the application of International Classification of Diseases (ICD) codes (see http://www.who.int/classifications/icd/en/). The evaluation of the importance of health outcomes should consider the strength of evidence for causality, as well as the condition's severity (related morbidity, disability and mortality), 
reversibility and frequency of its occurrence in the population. These features determine the public health importance of the health outcome.

The affected population has to be clearly identified with special attention to susceptible subgroups. Considering varying susceptibility in outcome assessment makes the evaluation of policy impact on health inequalities possible.

Health outcomes can be assessed in a qualitative or, if feasible, in a quantitative way. Qualitatively the direction of effect can be stated or the size of effect can be categorically described. However, a critical issue in the assessment of health outcomes is the possibility of quantification. It needs a decision on what kind of health measures, i.e. epidemiological frequency measures, to use as input and output data of the calculation process. In addition, consideration of the availability of valid baseline frequency data of the health condition and of dose/exposure-response functions that may apply dose-response coefficients or relative risks is indispensable for the success. Values of frequency measures of health conditions and of exposures must be available for the affected population. They usually derive from routine statistics, population-based registries or from surveys. Availability and validity of data is crucial in the process and therefore should be clearly described. The result of quantitative outcome assessment can be expressed in a frequency measure, like frequency of occurrence, morbidity, hospitalization and mortality (Example 1), or favourably in a complex measure of disease burden, like attributable death, potential years of life lost or disability adjusted life years (Example 2). The latter is an advantageous choice for expressing results of a risk assessment in a quantitative way, since it is a complex measure of disease burden combining effect on both morbidity and mortality. The cost-benefit analysis of policy introduction may also become possible based on the numerical expression of health outcomes. 
Example 1

The case study on the housing program of North Rhine-Westphalia assessed how the provision of barrier-free homes in the region would affect the incidence of falls, consequent hip fracture and death in the elderly population. The quantitative assessment was based on population statistics and information from the literature and estimated approximately 3000 to 8000 hip fractures and 600 to 1600 deaths due to hip fractures that could have been avoided in the year 2009, if all homes of people aged 65 years and older in the region were barrier-free (Gulis et al., 2013).

\section{Example 2}

The case study on the amendment of the Hungarian anti-smoking policy analysed the population health effect of banning smoking in all closed public places, workplaces and public transport vehicles. Data from the literature, national statistics and surveys were used to estimate the effect on disease burden. According to the conservative model that used $3 \%$ discounting and age-weighting, the point estimates of the long-term annual health gain were close to 1700 attributable death and 16000 disability adjusted life years in the Hungarian population of 10 million (Ádám et al., 2013).

\subsubsection{Cross-level issues}

A key issue in the assessment of health risks of policies is the quantification of health effects. Quantitative assessment can be perceived as the quantitative expression of expected changes in health outcome measures by using numerical information on how a policy affects health outcomes directly or through induced changes in exposure levels of risk factors. Quantitative 
expression of results has advantages to qualitative description. It is favoured in the decision making process, since it helps in prioritizing themes and considering cost-benefit relations, therefore it can effectively assist the bargain process. Constructing a logical algorithm from the policy proposal down to health outcomes can help in the clarification of the quantification process.

However, disadvantages of quantification should also be taken into consideration when applying such methods for assessment. A single estimate, even if favourable in decisionmaking, should not be the only disseminated result. A point estimate alone cannot reflect the complexity of the issue as well as the uncertainty of estimation. Health effects cannot be unconditionally added up as this may result in double counting, therefore the horizontal interrelation between various causal pathways at different levels should be assessed, too. Transparency is a universal prerequisite for the description of any assessment processes. Assessors should provide a clear explanation on the method of information search, evidence evaluation, prioritization of health determinants, risk factors and health outcomes, selection of applied measures and functions, data collection and validity assessment. An important factor of transparency is the description of uncertainties in the assessment process, too.

A crucial issue of professional correctness is the acknowledgement of limitations in the use of methodology. Admitting inability to assess health outcomes due to lack of data, functions, expert skills, etc., is a prerequisite of a transparent process description. Limitations also relate to the phenomenon of uncertainty. The statement that an outcome cannot be assessed implies infinite uncertainty.

Uncertainty is a natural attendant of predictions. The assessment of the impact of a policy, especially when it is prospective projection for the future, always involves uncertainty. It derives, among others, from the questionable strength of evidence and validity of data and functions applied. Numerical information is usually an estimate with inherited uncertainty due 
to random error of sampling, and it can be further enlarged by the presence of bias and by the extrapolation of information from one situation/population to another. Therefore the repeated statement that uncertainty exists has not much added value; rather its extent should be described in a qualitative or quantitative way. In the latter case, a range can be specified that functions as an interval estimate of the result. More about uncertainties and the way to communicate them could be found in published literature (WHO-IPCS, 2008; Knol, 2009). Realization of impact may need time. The description of probable latency of effects is important information in the decision-making process. There is a latency period between the planning and implementation of a policy, as well as a lag phase between policy implementation and development of health effects. The consideration of health outcomes dependent on time, i.e. differentiation between short and long term effects is a favourable product of an assessment.

To describe and assess strength of evidence on different levels of the full chain assessment, users are recommended to use guidance on levels of evidence developed by the National Institute for Health and Clinical Excellence of the United Kingdom (National Institute for Health and Clinical Excellence, 2005; Weightman et al., 2005).

\subsection{Checklist for the integrated assessment of health risks of policies}

The methodological tool, which is a product of consensus discussion based on individual national case studies, is summarized as a checklist that can be used together with the guidance for policy health risks assessment (Table 1). It identifies main issues to be addressed on each level of the impact chain and provides guidance on how to conduct the assessment in broad terms. 


\section{Discussion}

The Gothenburg consensus paper set the framework and the basic principles for health impact assessment (WHO, 1988). Since then, experience has accumulated and demands for the assessment of health effects of various proposals and initiatives have grown substantially (Veerman et al., 2005; Dannenberg et al., 2006; Dannenberg et al., 2008). Several guidelines have been developed with the aim to unify methodology and aid users in their efforts to predict impact on health (Scott-Samuel et al., 2001; Taylor and Blair-Stevens, 2002; Health Canada, 2004; Abrahams el al., 2004; Cole et al., 2005; Quigley et al., 2006; Harris et al., 2007; Metcalfe et al., 2009; Bhatia et al, 2010; Bathia, 2010; Human Impact Partners, 2011; Chadderton et al., 2013). Methods for the quantitative assessment of health outcomes have also been formulated, typically providing a model for the characterization of risk factor-health outcome relation (Veerman et al., 2005; Boshuizen et al., 2012). They either apply an algorithm to predict risk in an epidemiological approach or use classical toxicological functions. The latter is typical for the assessment of health effects related to environmental exposures (Basham, 2001; Samoli et al., 2008; Kheirbek et al., 2013). Framework for the integrated quantitative assessment of health risks in environmental health impact assessment has been developed, too (Briggs, 2008). However, a universal methodological guideline for the assessment of the complex impact structure of policies that typically include not only environmental but also socio-economic determinants of health has been lacking. The intention of the RAPID project was to formulate a methodological tool for those who want to carry out comprehensive assessment of health risks related to policy proposals. It can be used either as a standalone tool or within the risk appraisal part of a HIA. Although impact assessment is required within the European Commission as a preparatory action for EC decisions but it discusses health issues rather rarely. The impact assessment of EU policies is mostly oriented towards economy, competitiveness and social issues (employment, 
education). The presented tool is designed to serve the implementation of the "Health in all policies" principle on national and international level alike, as it allows for the comprehensive systematic assessment of health effects of policies and the identification of the role of relevant sectors within policy development. To reach the potential users, information on the development of the tool was disseminated by the project website (RAPID, 2012), via a series of workshops at European public health conferences as well as at national workshops organised for risk assessor in the time period of 2009-2012.

The most important limitations of the guidance are the lack of experience from external testing, its "broadness", and its sensitivity to cultural issues and understanding of terminology. The guidance was developed as a consensus document based on national case studies, the EU case study and consultation via national workshops. Nevertheless, experiences from testing the completed guidance on different policy topics could add valuable information to its further improvement. We would like to encourage readers to use it and provide feedback. The broadness of the guidance is given by the topic; policies are usually complex so should be the guidance for risk assessment. In different countries public health has different meanings and so do the terms, such as determinants of health and risk factors which are often used interchangeably. This poses the guidance to a kind of cultural sensitivity, e.g. terms and steps can be diversely interpreted based on the understanding of their meaning.

Despite of the limitations, the broad range of topics addressed to develop the guidance ensures its wide applicability. The case studies included tobacco and energy policy, housing subsidy program, air pollution legislation, policy on alcohol, wine production, road safety, X-ray computed tomography use and influenza pandemics interventions. Details about the case studies can be found in the book by Gulis et al. (2013) that describes the achievements of the RAPID project. 
The comprehensive assessment of health impact of various policies requires the mapping of the full impact scheme, following each level of the causal chain from the policy proposal through related health determinants and risk factors to health outcomes. The prioritisation of factors on each level in a systematic transparent way, the consideration of evidence and feasibility of assessment including advantages and disadvantages of quantification, the assessment of horizontal interactions between impact pathways, the evaluation of the latency period for the realization of health effects and the description of uncertainty of estimates are the major issues identified and addressed by our work. The checklist offers a logical framework that lists up important issues to be considered during the assessment process and gives advice on implementation. The guidance provides detailed explanation about the critical aspects of assessment that helps using the checklist. However, this combined tool is not a cookbook. It is intended to be used by those who have previous experience with risk assessment and HIA, although it can offer assistance in getting started for those who are new in the field, too. 


\section{Acknowledgements}

This work was completed in the framework of the Risk Assessment from Policy to Impact

Dimension (RAPID) project funded by the European Commission, Health \& Consumer

Protection Directorate-General (grant agreement number 20081105). 


\section{References}

Abrahams D, Pennington A, Scott-Samuel A, Doyle C, Metcalfe O, den Broeder L, el al. European Policy Health Impact Assessment: A Guide. Brussels: Health and Consumer Protection Directorate General, European Commission; 2004.

Ádám B. A model of health. Public Service Review: Health and Social Care 2012;32. Available at: http://www.publicservice.co.uk/article.asp?publication=Health and Social Care\&id=568\&content_name=European Health and Social Care Focus\&article=20175. (accessed 29 July 2013)

Ádám B, Molnár Á, Gulis G, Ádány R. Integrating quantitative risk appraisal in health impact assessment: analysis of the novel smoke-free policy in Hungary. Eur J Pub Health $2013 ; 23: 211-7$

Barton H, Grant M: A health map for the local human habitat, Journal of the Royal Society for the Promotion of Public Health, 126(6), p. 252-261

Basham J. Application of COMEAP dose-response coefficients within a regulatory health impact assessment methodology. Committee on the Medical Effects of Air Pollutants. J Public Health Med 2001;23:212-8.

Bathia R. A Guide for Health Impact Assessment. Sacramento: California Department of Public Health; 2010.

Bhatia R, Branscomb J, Farhang L, Lee M, Orenstein M, Richardson M. Minimum Elements and Practice Standards for Health Impact Assessment, Version 2. Oakland: North American HIA Practice Standards Working Group; 2010.

Briggs DJ. A framework for integrated environmental health impact assessment of systemic risks. Environ Health 2008;7:61. 
Boshuizen HC, Lhachimi SK, van Baal PH, Hoogenveen RT, Smit HA, Mackenbach JP, et al. The DYNAMO-HIA model: an efficient implementation of a risk factor/chronic disease Markov model for use in Health Impact Assessment (HIA). Demography 2012;49:125983.

Chadderton C, Elliott E, Green L, Lester J, Williams G. Health Impact Assessment. A practical guide. Cardiff: Wales Health Impact Assessment Support Unit; 2013.

Cole BL, Shimkhada R, Fielding JE, Kominski G, Morgenstern H. Methodologies for realizing the potential of health impact assessment. Am J Prev Med 2005;28:382-9.

Dahlgren G, Whitehead M. Policies and strategies to promote social equity in health. Stockholm: Institute for Future Studies; 1991.

Dannenberg AL, Bhatia R, Cole BL, Dora C, Fielding JE, Kraft K, et al. Growing the field of health impact assessment in the United States: an agenda for research and practice. Am J Public Health 2006;96:262-70.

Dannenberg AL, Bhatia R, Cole BL, Heaton SK, Feldman JD, Rutt CD. Use of health impact assessment in the U.S. 27 case studies, 1999-2007. Am J Prev Med 2008;34:241-56.

Davenport C, Mathers J, Parry J. Use of health impact assessment in incorporating health considerations in decision making. J Epidemiol Community Health 2006;60:196-201.

Gulis G, Mekel O, Ádám B, Cori L (eds). Assessment of Population Health Risks of Policies. New York: Springer Science + Business; 2013.

Harris P, Harris-Roxas B, Harris E, Kemp L. Health Impact Assessment: A Practical Guide. Sydney: Centre for Health Equity Training, Research and Evaluation, University of New South Wales; 2007.

Health Canada. Canadian handbook on health impact assessment. Ottawa: Health Canada, 2004. 
Human Impact Partners. A Health Impact Assessment Toolkit: A Handbook to Conducting HIA, 3rd ed. Oakland: Human Impact Partners; 2011.

Kheirbek I, Wheeler K, Walters S, Kass D, Matte T. PM2.5 and ozone health impacts and disparities in New York City: sensitivity to spatial and temporal resolution. Air Qual Atmos Health 2013 Jun;6:473-86.

Knol AB, Petersen AC, van der Sluijs JP, Lebret E. Dealing with uncertainties in environmental burden of disease assessment. Environ Health 2009;8:21.

Lalonde M. A new perspective on the health of Canadians. Ottawa: Government of Canada, 1974. Available at: http://www.phac-aspc.gc.ca/ph-sp/pdf/perspect-eng.pdf. (accessed 29 July 2013)

Lhachimi SK, Nusselder WJ, Boshuizen HC, Mackenbach JP. Standard tool for quantification in health impact assessment: a review. Am J Prev Med 2010;38:78-84.

Lhachimi SK, Nusselder WJ, Smit HA, van Baal P, Baili P, Bennett K, et al. DYNAMOHIA--a Dynamic Modeling tool for generic Health Impact Assessments. PLoS One 2012;7:e33317.

Mannheimer LN, Gulis G, Lehto J, Ostlin P. Introducing Health Impact Assessment: an analysis of political and administrative intersectoral working methods. Eur J Public Health 2007; 17:526-31.

Manuel DG, Rosella LC, Hennessy D, Sanmartin C, Wilson K. Predictive risk algorithms in a population setting: an overview. J Epidemiol Community Health 2012;66:859-65.

McCarthy M, Biddulph JP, Utley M, Ferguson J, Gallivan S. A health impact assessment model for environmental changes attributable to development projects. J Epidemiol Community Health 2002;56:611-6.

Mesa-Frias M, Chalabi Z, Foss AM. Assessing framing assumptions in quantitative health impact assessments: a housing intervention example. Environ Int 2013;59:133-40. 
Metcalfe O, Higgins C, Lavin T. Health Impact Assessment Guidance. Dublin: Institute of Public Health in Ireland; 2009.

National Institute for Health and Clinical Excellence. Guideline Development Methods. Chapter 7 Reviewing and grading the evidence. London: NIHCE; 2005.

Quigley R, den Broeder L, Furu P, Bond A, Cave B, Bos R. Health Impact Assessment: International Best Practice Principles. Fargo: International Association for Impact Assessment; 2006.

Risk Assessment from Policy to Impact Dimension. Project website. Available at: http://www.sdu.dk/en/RAPID.aspx (accessed 10 November 2013)

Samoli E, Peng R, Ramsay T, Pipikou M, Touloumi G, Dominici F, et al. Acute effects of ambient particulate matter on mortality in Europe and North America: results from the APHENA study. Environ Health Perspect 2008;116:1480-6.

Scott-Samuel A, Birley M, Ardern K. The Merseyside Guidelines for Health Impact Assessment. 2nd ed. Liverpool: International Health Impact Assessment Consortium; 2001.

Ståhl T, Wismar M, Ollila E, Lahtinen E, Leppo K. Health in All Policies. Prospects and potentials. Helsinki: Ministry of Social Affairs and Health; 2006.

Steenland K, Armstrong B. An overview of methods for calculating the burden of disease due to specific risk factors. Epidemiology 2006;17:512-9.

Taylor L, Blair-Stevens C. Introducing health impact assessment (HIA): Informing the decision-making process. London: Health Development Agency; 2002.

Utley M, Gallivan S, Biddulph J, McCarthy M, Ferguson J. ARMADA—a computer model of the impact of environmental factors on health. Health Care Manag Sci 2003;6:137-46.

Veerman JL, Barendregt JJ, Mackenbach JP. Quantitative health impact assessment: current practice and future directions. J Epidemiol Community Health 2005;59:361-70. 
Weightman A, Ellis S, Cullum A, Sander L, Turley R. Grading evidence and recommendations for public health interventions: developing and piloting a framework. London: Health Development Agency; 2005.

WHO European Centre for Health Policy. Health impact assessment: main concepts and suggested approach. Gothenburg Consensus Paper. Brussels: WHO European Centre for Health Policy; 1999.

World Health Organization. Adelaide Recommendations on Healthy Public Policy. Geneva: WHO; 1988.

World Health Organization. Ottawa Charter for Health Promotion. Ottawa: WHO; 1986.

World Health Organization. Health Promotion Glossary. Geneva: WHO; 1998.

World Health Organization-International Programme on Chemical Safety. Guidance document on characterizing and communicating uncertainty in exposure assessment. Harmonization Project Document No. 6 (Part 1). Geneva: WHO; 2008.

World Health Organization, Government of South Australia. Adelaide Statement on Health in All Policies: moving towards a shared governance for health and well-being. Geneva: WHO; 2010. 